\title{
XÁC ĐỊNH HÀM LƯợNG SIBUTRAMIN VÀ PHENOLPHTHALEIN TRONG TRÀ, CÀ PHÊ, SỮA GIẢM CÂN BẰNG PHƯơNG PHÁP SẮC KÝ LỎNG SIÊU HIỆ NĂNG GHÉP NỐl KHỐI PHỔ UPLC-MS/MS
}

\author{
Trương Văn Thân, Nguyễn Thành Duy*, Lý Tuấn Kiệt, \\ Hoàng Ngọc Vinh, Chu Vân Hải
}

Trung tâm dịch vu phân tích thí nghiệm Tp. Hồ Chí Minh

(Ngày đến tòa soạn: 2/7/2019; Ngày sửa bài sau phản biện: 3/9/2019;

Tóm tắt

Ngày chấp nhận đăng: 12/9/2019)

Một phương pháp nhanh chóng, đơn giản và hiệu quả để xác định sibutramin và phenolphthalein trong trà, cà phê, sữa giảm cân và được phát triển bằng phương pháp sắc ký lỏng siêu hiệu năng kết hợp với đầu dò khối phổ (mass spectrometry). Kỹ thuật QuEChERS được sử dụng để làm sạch các nền mẫu. Dịch chiết sau đó được tách trên cột sắc ký lỏng $\mathrm{C} 18$ bằng hỗn hợp nước loại ion và acetonitril, với $0,1 \%$ acid formic. Hiệu suất thu hồi ở nồng độ 50 $\mu \mathrm{g} / \mathrm{kg}$ đạt được từ $90,1-105,8\left(\mathrm{RSD}_{\mathrm{R}} 4,04-4,24 \%\right)$ đối với trà giảm cân và từ $91,9-100,2$ $\left(\operatorname{RSD}_{R} 3,20-4,71 \%\right)$ đối với cà phê giảm cân và $86,5-100,5 \%\left(\operatorname{RSD}_{R} 5,17-5,49 \%\right)$ đối với sữa giảm cân. Giới hạn định lượng $30 \mu \mathrm{g} / \mathrm{kg}$ cho cả 3 nền mẫu.

Tù̀ khóa: Sibutramin, phenolphthalein, thưc phẩm giảm cân, sắc ký lỏng siêu hiệu năng ghép khối phổ UPLC-MS/MS.

\section{1. ĐẠTT VẤN ĐỀ}

Béo phì được công nhận là một vấn đề sức khỏe cộng đồng trên toàn thế giới. Bổ sung vào chế độ ăn uống đã được sử dụng như phương pháp điều trị thay thế cho bệnh béo phì. Tuy nhiên, một số chất bổ sung giảm cân đáng quan tâm hiện nay là sibutramin và phenolphthalein. Sibutramin là một hợp chất được sử dụng để giảm sự thèm ăn, chất này có thể gây nguy cơ tăng huyết áp, đau tim cho người bình thường và có thể gây nguy hiểm cho những người có tiền sử bệnh về động mạch vành, đau tim, loạn nhịp tim hoặc đột quỵ. Còn phenolphthalein là một hóa chất thường được sử dụng trong việc đo độ kiềm/acid $(\mathrm{pH})$ của dung dịch dựa vào khả năng đổi màu của nó. Chất này đã từng được sử dụng trong điều trị táo bón nhưng do các nghiên cứu cho thấy nó có khả năng là chất gây ung thư (carcinogen) [1]. Điều này gây ảnh hưởng sức khỏe cho người tiêu dùng.

Trong nghiên cứu này, chúng tôi đã xây dựng quy trình phân tích sibutramin và phenolphthalein nhanh chóng, đơn giản và hiệu quả kết hợp kỹ thuật chiết và tinh sạch mẫu bằng phương pháp QuEChERS trong trà, cà phê, sữa giảm cân. Sau đó, dịch chiết được phân tích trên hệ thống sắc ký lỏng siêu hiệu năng kết hợp với đầu dò khối phổ (UPLC-MS/MS).

\section{2. ĐỐI TƯỢNG VÀ PHƯƠNG PHÁP NGHIÊN CỨU}

\section{1. Đối tượng nghiên cứu}

Các mẫu thực phẩm giảm cân bao gồm trà, cà phê, sữa giảm cân do khách hàng gửi được phân tích tại trung tâm dịch vụ phân tích thí nghiệm Tp. Hồ Chí Minh (CASE).

\subsection{Phương pháp nghiên cứu}

2.2.1. Thiết bị, dụng cụ, hóa chất

\footnotetext{
*Điện thoại:0909610128Ｅmail:duynt@case.vn
}

42 | Tạp chí KIỂM NGHIẸM VÀ AN TOÀN THỰC PHẨM (Số 4-2019) 
2.2.1.1 Thiết bị, dụng cu

- Thiết bị sử dụng là hệ UPLC Endura và khối phổ TSQ Endura Triplequad của hãng Thermo Scientific.

- Cột phân tích sắc ký pha đảo InertSustain C18 HP $3 \mu \mathrm{m}(100$ x 2,1) mm và cột bảo vệ.

- Các dụng cụ và thiết bị phụ trợ khác trong phòng thí nghiệm.

\subsubsection{Hóa chất}

- Acetonitril và methanol loại LC-MS, acid formic ( $\geq 99 \%)$, acid acetic ( $\geq 99 \%)$

- Chuẩn phenolphthalein (Product No.: 107233. Lot No.: K49210033) được cung cấp Merck millipore.

- Chuẩn sibutramin (Product No.: S-011-1ML. Lot No.: FE03201802) của hãng SigmaAldrich.

- Bột chiết pha rắn Q-sep d-SPE (150 mg $\mathrm{MgSO}_{4}, 50 \mathrm{mg} \mathrm{C}$ 18), d-SPE $\left(150 \mathrm{mg} \mathrm{MgSO}_{4}, 50\right.$ $\mathrm{mg} \mathrm{C} 18,50 \mathrm{mg}$ PSA), hỗn hợp muối (4 $\left.\mathrm{g} \mathrm{MgSO}_{4}, 1 \mathrm{~g} \mathrm{NaCl}\right)$ của $\mathrm{CNW}$.

\subsubsection{Tiến hành}

\subsubsection{Chuẩn bị chất chuẩn}

Dung dịch chuẩn gốc nồng độ 1000 ppm $(\mathrm{mg} / \mathrm{L})$ trong dung môi methanol. Dung dịch chuẩn gốc được lưu nhiệt độ $\mathrm{t}^{\circ}<-18^{\circ} \mathrm{C}$. Chuẩn hỗn hợp trung gian nồng độ $500 \mathrm{ppb}(\mu \mathrm{g} / \mathrm{L})$ được pha từ dung dịch chuẩn gốc trong methanol và được sử dụng để xây dựng đường chuẩn trên mẫu.

\subsubsection{Xủ lý mẫu}

Mẫu trà, cà phê, sữa giảm cân được đồng nhất. Cân chính xác khoảng $1 \mathrm{~g}$ cho vào ống ly tâm, thêm $5 \mathrm{~mL} \mathrm{H}_{2} \mathrm{O}$, vortex 1 phút. Sau đó, thêm $10 \mathrm{~mL}$ acid acetic $1 \%$ trong acetonitril, vortex 1 phút, siêu âm 30 phút, nhiệt độ thường. Thêm hỗn hợp muối $\left(\mathrm{MgSO}_{4} 4 \mathrm{~g}+\mathrm{NaCl} 1 \mathrm{~g}\right)$, lắc mạnh bằng tay, vortex 1 phút, ly tâm 9000 vòng/phút $\left(20^{\circ} \mathrm{C}, 5\right.$ phút). Rút $1 \mathrm{~mL}$ lớp trên cho vào các ống chiết pha rắn d-SPE $\left(150 \mathrm{mg} \mathrm{MgSO}_{4}, 50 \mathrm{mg} \mathrm{C18}\right)$ để làm sạch mẫu, vortex 1 phút, ly tâm 10000 vòng/phút $\left(20^{\circ} \mathrm{C}, 5\right.$ phút). Rút $200 \mu \mathrm{L}$ dung dịch thổi khô. Định mức $400 \mu \mathrm{L}$ $\mathrm{MeOH} / \mathrm{H}_{2} \mathrm{O}(1 / 1)$ và được tiêm vào hệ thống $\mathrm{UHPLC} / \mathrm{MS} / \mathrm{MS}$.

\subsubsection{3. Điều kiện sắc ký}

Để xây dựng quy trình phân tích đồng thời sibutramin và phenolphthalein, các điều kiện sắc ký dưới đây đã được chúng tôi nghiên cứu và sử dụng:

- Cột InertSustain C18 HP $3 \mu \mathrm{m}(100$ x 2,1) mm.

- Tốc độ dòng pha động: $0,3 \mathrm{~mL} /$ phút

- Pha động:

Kênh A: $\mathrm{HCOOH} \mathrm{0,1 \%} \mathrm{(v/v)} \mathrm{trong} \mathrm{nước;}$

Kênh B: acetonitril;

Gradient: 0 - 5 phút: 5\% B - 100\% B , 5 - 7 phút: $100 \%$ B, 7 - 10 phút: $5 \%$ B

- Thể tích bơm mẫu: $5 \mu \mathrm{L}$

- Thông số đầu dò MS: Ion source type: H-ESI, positive ion: ESI (+), spay voltage: 3500V, sheath gas (Arb): 45 , aux gas (Arb): 13 , sweep gas (Arb): 1 , ion transfer tube term: $342^{\circ} \mathrm{C}$, vaporizer term: $358^{\circ} \mathrm{C}$.

\section{KẾT QUẢ VÀ BÀN LUẦN}

\subsection{Tối ưu thông số đầu dò và điều kiện sắc ký}

Qua tham khảo các tài liệu $[3,4,5]$, và khảo sát tại phòng thí nghiệm chúng tôi dùng dung dịch chuẩn 100 ppb để tìm các thông số tối ưu cho khối phổ như tìm ion mẹ, ion con, năng lượng 
va chạm và RF Lens. Sau khi thực hiện ta có thông số đầu dò khối phổ được thiết lập theo bảng 1 và sử dụng cột tách $\mathrm{C} 18$ với pha động theo chương trình rửa giải gradient gồm hai kênh chứa $\mathrm{HCOOH} 0,1 \%$ trong nước (kênh $\mathrm{A})$ và $\mathrm{ACN}(\mathrm{kênh} \mathrm{B})$ cho hiệu quả tách tốt nhất sibutramin và phenolphthalein.

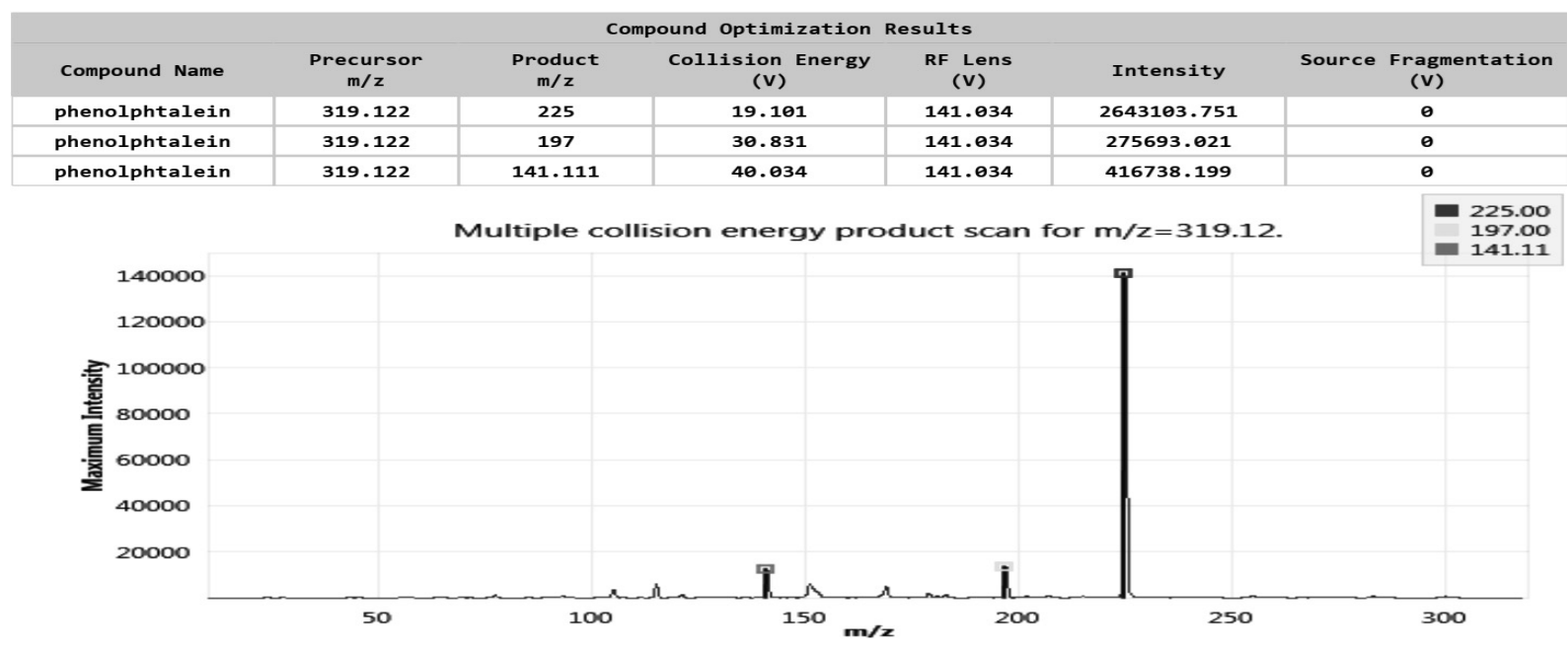

Hinh 1. Các thông số tối ưu khối phổ của sibutramin

\begin{tabular}{|c|c|c|c|c|c|c|}
\hline \multicolumn{7}{|c|}{ Compound Optimization Results } \\
\hline Compound Name & $\begin{array}{c}\text { Precursor } \\
\mathrm{m} / \mathrm{z}\end{array}$ & $\begin{array}{l}\text { Product } \\
\mathrm{m} / \mathrm{z}\end{array}$ & $\begin{array}{c}\text { Collision Energy } \\
\text { (v) }\end{array}$ & $\begin{array}{l}\text { RF Lens } \\
\text { (v) }\end{array}$ & Intensity & $\begin{array}{c}\text { Source Fragmentation } \\
\text { (v) }\end{array}$ \\
\hline phenolphtalein & 319.122 & 225 & 19.101 & 141.034 & 2643103.751 & $\theta$ \\
\hline phenolphtalein & 319.122 & 197 & 30.831 & 141.034 & 275693.021 & $\theta$ \\
\hline phenolphtalein & 319.122 & 141.111 & 40.034 & 141.034 & 416738.199 & $\theta$ \\
\hline
\end{tabular}

Multiple collision energy product scan for $m / z=319.12$.

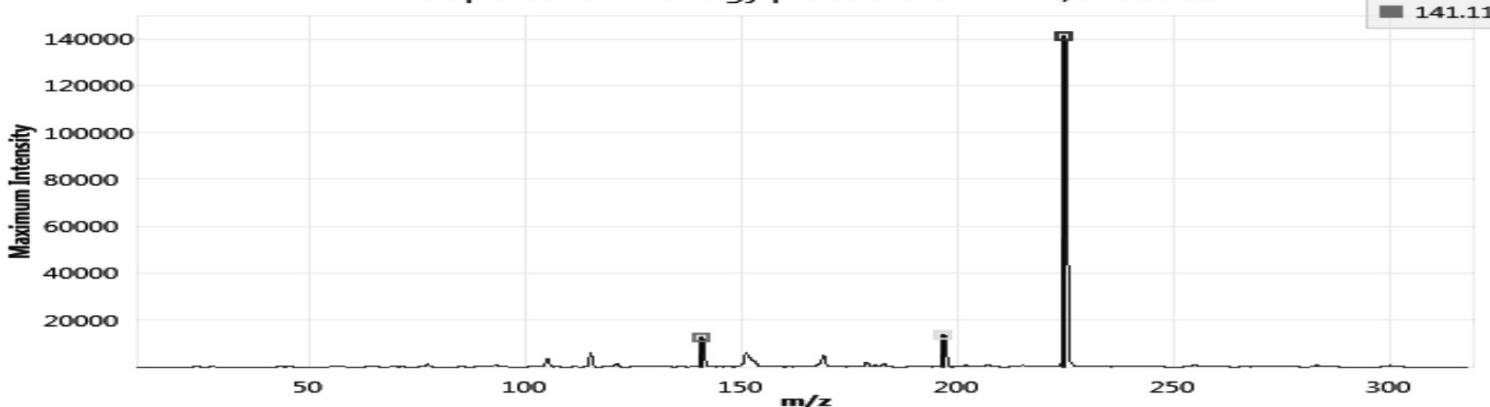

Hinh 2. Các thông số tối ưu khối phổ của phenolphthalein

Bảng 1. Các thông số khối phổ ở chế độ SRM

\begin{tabular}{|c|c|c|c|c|c|c|}
\hline Chất phân tích & $\begin{array}{c}\text { Thòi gian } \\
\text { luu } \\
\text { (phút) }\end{array}$ & Mode & $\begin{array}{c}\text { Ion me } \\
(m / z)\end{array}$ & $\begin{array}{c}\text { Ion con } \\
(m / z)\end{array}$ & $\begin{array}{l}\text { Năng luọng } \\
\text { va chạm }(V)\end{array}$ & $\begin{array}{c}R F \\
\text { Lens } \\
(V)\end{array}$ \\
\hline \multirow{2}{*}{ Sibutramin } & \multirow{2}{*}{4,42} & \multirow{2}{*}{$(+)$} & 280,1 & $139,0(\mathrm{C})$ & 14 & 106 \\
\hline & & & 280,1 & $125,0(\mathrm{Q})$ & 24 & 106 \\
\hline \multirow{2}{*}{ Phenolphthalein } & \multirow{2}{*}{4,82} & \multirow{2}{*}{$(+)$} & 319,1 & $197,0(\mathrm{C})$ & 30 & 141 \\
\hline & & & 319,1 & $225,0(\mathrm{Q})$ & 19 & 141 \\
\hline
\end{tabular}

\subsection{Lựa chọn hỗn họ̣p bột chiết pha rắn d-SPE}

Do đối tượng nghiên cứu của đề tài là nền mẫu trà, cà phê, sữa giảm cân là nền mẫu phức 
tạp, cần quy trình xử lý mẫu để làm sạch tạp chất nhằm giảm hiện tượng ảnh hưởng nền, tăng hiệu quả trích ly mẫu, chúng tôi nghiên cứu 2 loại bột chiết pha rắn bao gồm d-SPE (150 mg $\left.\mathrm{MgSO}_{4}, 50 \mathrm{mg} \mathrm{C} 18\right)$ và $\mathrm{d}-\mathrm{SPE}\left(150 \mathrm{mg} \mathrm{MgSO}_{4}, 50 \mathrm{mg} \mathrm{C} 18,50 \mathrm{mg} \mathrm{PSA}\right)$ để làm sạch mẫu trước khi đưa vào thiết bị UPLC/MS/MS.

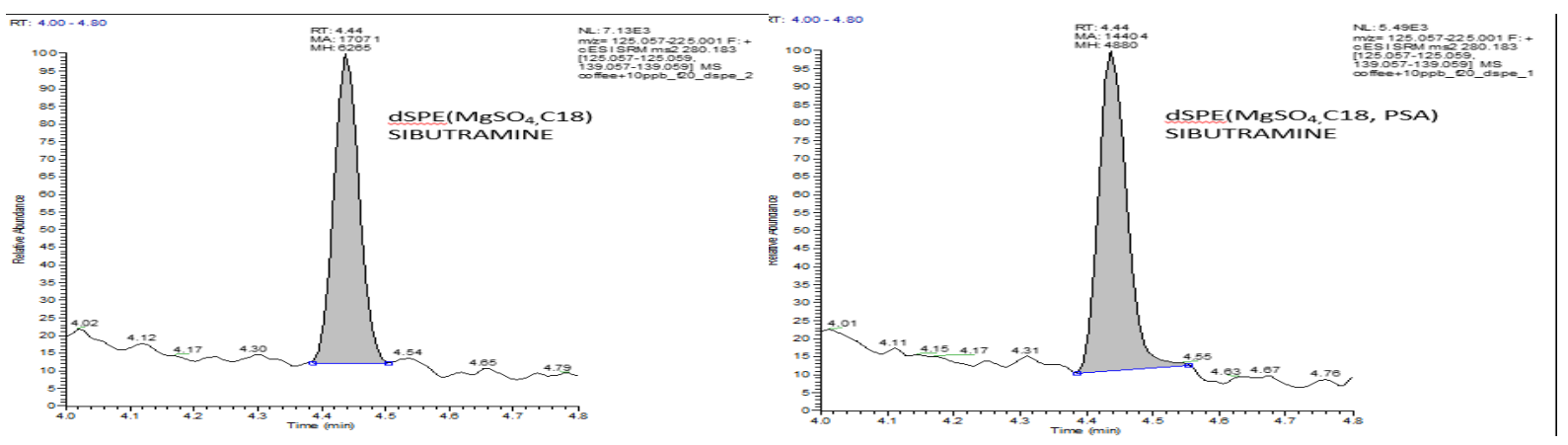

Hình 3. Mẫu cà phê thêm $10 \mathrm{ng} / \mathrm{mL}$ chuẩn sibutramin so sánh bột chiết pha rắn

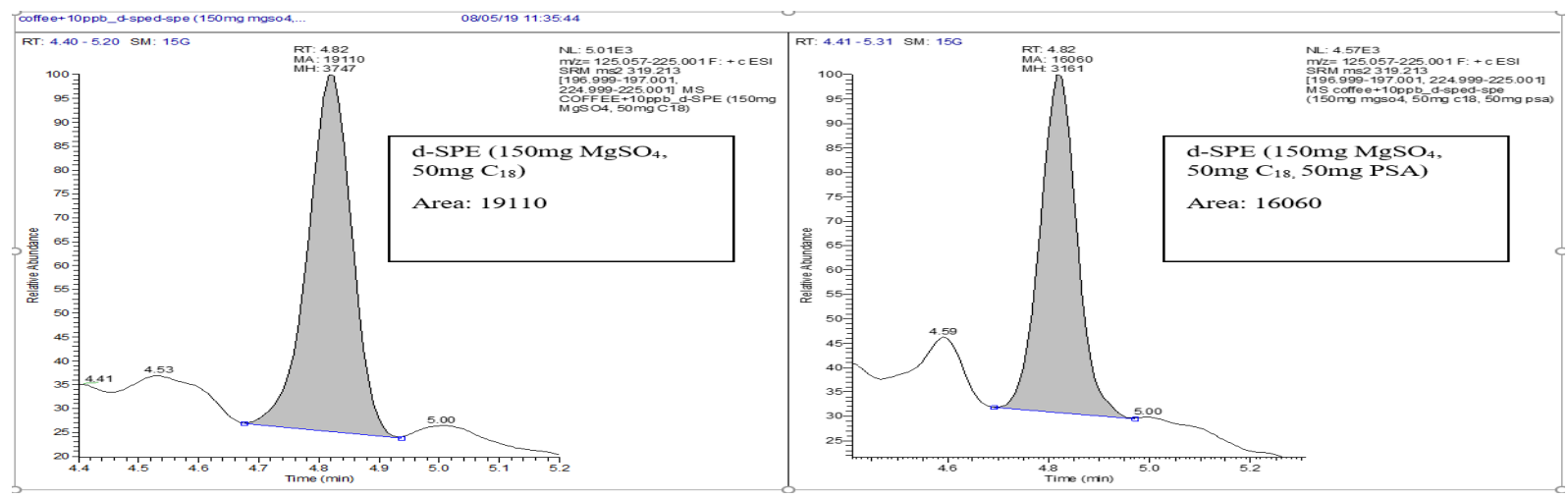

Hình 4. Mẫu cà phê thêm $10 \mathrm{ng} / \mathrm{mL}$ chuẩn phenolphthalein so sánh bột chiết pha rắn

Kết quả sắc ký đồ hình 2 cho thấy sibutramin và phenolphthalein trong mẫu sử dụng d-SPE (150mg $\mathrm{MgSO}_{4}, 50 \mathrm{mg} \mathrm{C18)}$ cho tín hiệu tốt hơn sibutramin và phenolphthalein trong mẫu sử dụng bột có chứa thêm $50 \mathrm{mg}$ PSA.

Như vậy quy trình xử lý mẫu 2.2.2.2 được lựa chọn thẩm định phương pháp sibutramin và phenolphthalein trong ba nền mẫu trà, cà phê, sữa giảm cân.

\subsection{Thẩm định phương pháp}

\subsection{1. Đường chuẩn sibutramin và phenolphthalein trên các nền mẫu}

Đường chuẩn được xây dựng dựa vào các điểm có nồng độ: $10 \mathrm{ng} / \mathrm{mL} ; 25 \mathrm{ng} / \mathrm{mL} ; 50$ $\mathrm{ng} / \mathrm{mL} ; 75 \mathrm{ng} / \mathrm{mL} ; 100 \mathrm{ng} / \mathrm{mL}$.

Nền cà phê giảm cân

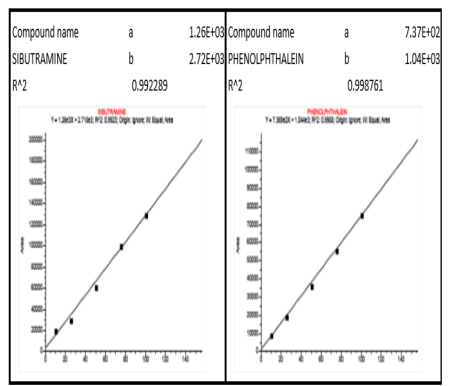

Nền trà giảm cân

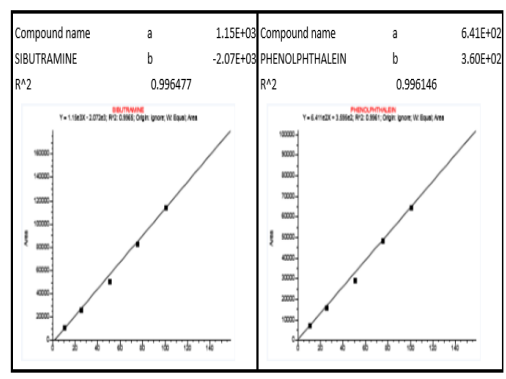

Nền sữa giảm cân

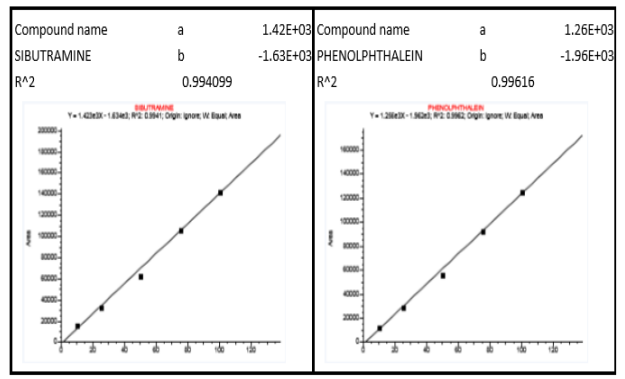

Hình 5. Đường chuẩn sibutramin và phenolphthalein trong các nền cà phê, trà và sũa giảm cân 
3.3.2. Độ thu hồi, độ lạp lại và độ tái lập, độ không đảm bảo đo

Độ lặp lại của phương pháp được thể hiện thông qua độ lệch chuẩn lặp lại $\left(\mathrm{RSD}_{\mathrm{r}}\right)$, còn độ tái lập được đánh giá thông qua độ lệch chuẩn tái lập $\left(\mathrm{RSD}_{\mathrm{R}}\right)$ của các kết quả nghiên cứu ở hai ngày thực hiện phân tích khác nhau.

Bảng 2. Kết quả độ thu hồi, độ lặp lại và độ tái lập, độ không đảm bảo đo

\begin{tabular}{|c|c|c|c|c|c|c|}
\hline Chỉ tiêu & $\begin{array}{l}\text { Nền } \\
\text { mấu }\end{array}$ & $\begin{array}{c}\text { Nồng độ thêm } \\
\text { mẫu }(\mu g / k g)\end{array}$ & $\begin{array}{c}\text { Độ thu } \\
\text { hồi } \%, \\
n=12\end{array}$ & $\begin{array}{c}R S D_{r} \%, \\
n=6\end{array}$ & $\begin{array}{c}R S D_{R} \%, \\
n=12\end{array}$ & $\begin{array}{l}\text { Độ không đảm } \\
\text { bảo đo } U_{\text {exp }}(\%)\end{array}$ \\
\hline \multirow{6}{*}{ Sibutramin } & \multirow{2}{*}{ Trà } & 30 & 101,1 & 10,2 & 11,5 & 20,5 \\
\hline & & 50 & 96,1 & 4,1 & 5,1 & 11,3 \\
\hline & \multirow{2}{*}{ Cà phê } & 30 & 99,2 & 5,4 & 6,2 & 12,6 \\
\hline & & 50 & 97,5 & 4,1 & 4,7 & 10,6 \\
\hline & \multirow{2}{*}{ Sữa } & 30 & 87,1 & 3,2 & 3,6 & 26,8 \\
\hline & & 50 & 90,9 & 4,5 & 5,5 & 21,2 \\
\hline \multirow{6}{*}{ Phenolphthalein } & \multirow{2}{*}{ Trà } & 30 & 94,9 & 7,2 & 8,5 & 17,6 \\
\hline & & 50 & 99,5 & 4,4 & 5,7 & 8,5 \\
\hline & \multirow{2}{*}{ Cà phê } & 30 & 98,6 & 4,2 & 3,9 & 8,3 \\
\hline & & 50 & 95,3 & 3,8 & 3,2 & 11,4 \\
\hline & \multirow{2}{*}{ Sữa } & 30 & 90,8 & 2,5 & 2,9 & 19,2 \\
\hline & & 50 & 95,2 & 4,8 & 5,2 & 14,1 \\
\hline
\end{tabular}

\subsubsection{Giới hạn định luợng}

Theo SANCO/12571/2013 [7], MQL là nồng độ thêm chuẩn ở nồng độ nhỏ nhất mà phương pháp có thể xác định với độ lặp lại, độ tái lặp nhỏ hơn $20 \%$, hiệu suất thu hồi đạt từ $80-120 \%$, phương pháp có ý nghĩa khi MQL nên bằng hoặc nhỏ hơn giới hạn chất cấm nhỏ nhất cho phép. Trong phần nghiên cứu này, mẫu trà, cà phê, sữa giảm cân không có chất phân tích thêm chuẩn ở nồng độ $30 \mu \mathrm{g} / \mathrm{kg}$ để xác định giá trị MQL. Kết quả phân tích thu được tất cả các chất đều có hiệu suất thu hồi trên $80 \%$, độ tái lặp nhỏ hơn $20 \%$. Như vậy, MQL của phương pháp đối với các nền mẫu là $30 \mu \mathrm{g} / \mathrm{kg}$.

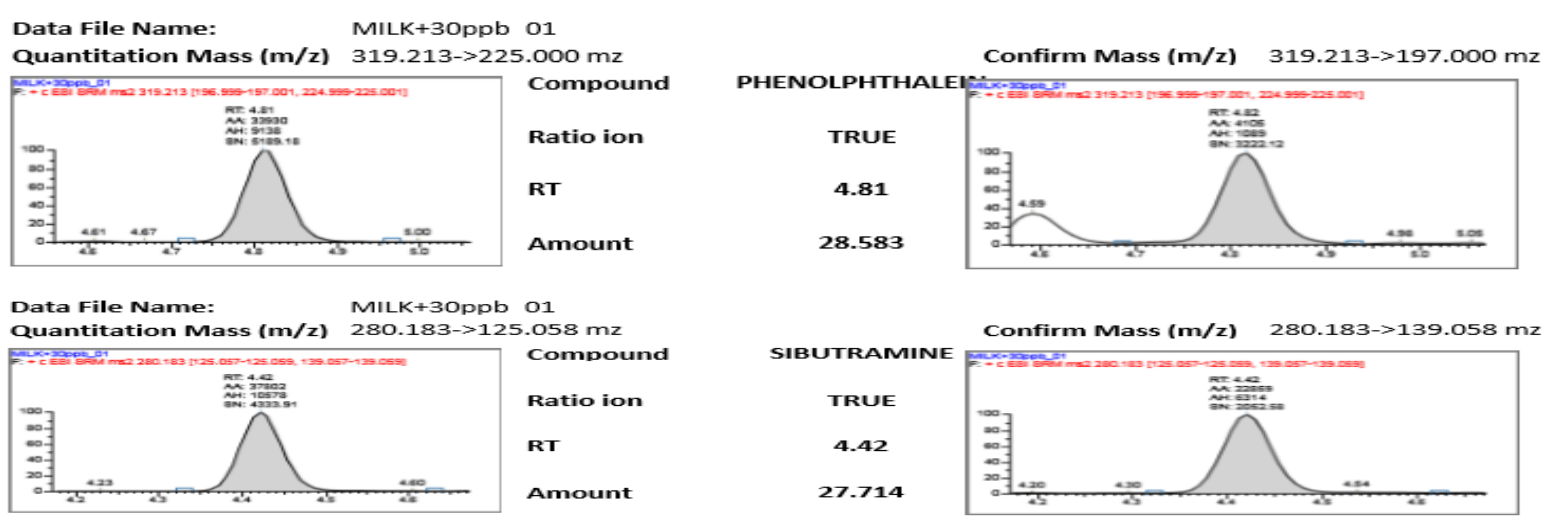

Hình 3.3.3 a. Sắc ký đồ mẫu sũa giảm cân thêm $30 \mu \mathrm{g} / \mathrm{kg}$ chuẩn sibutramin và phenolphthalein 

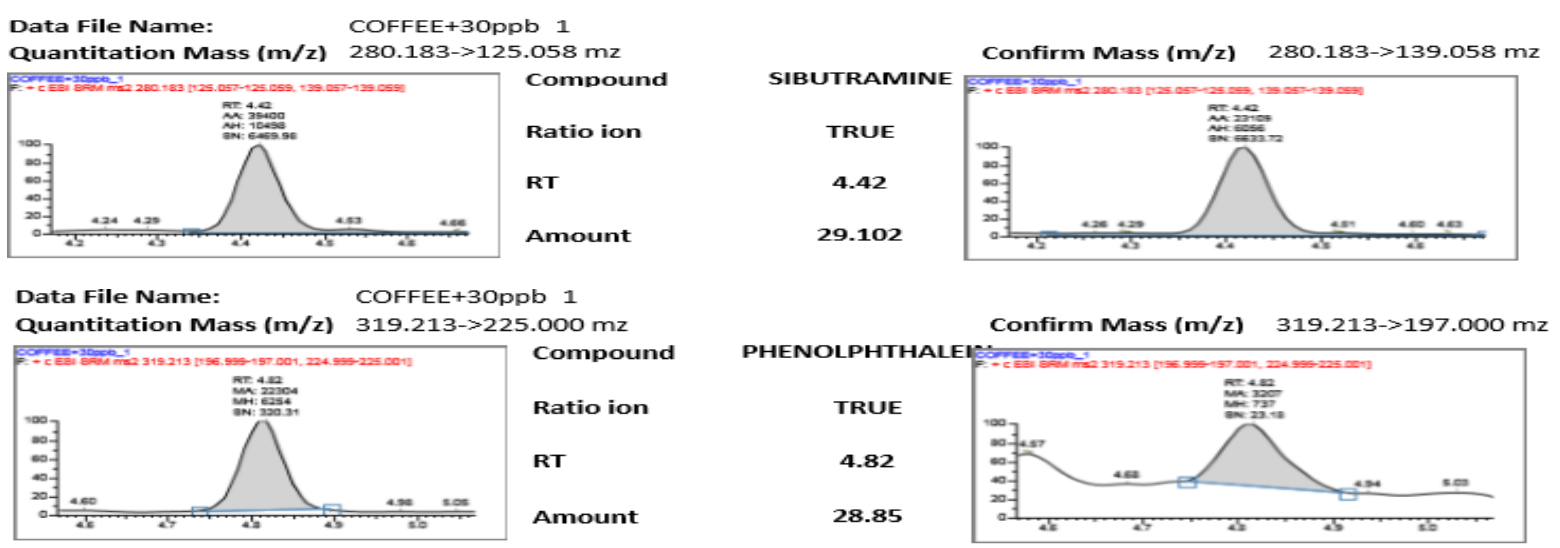

Hinh 3.3.3 b. Sắc ký đồ mẫu cà phê giảm cân thêm $30 \mu \mathrm{g} / \mathrm{kg}$ chuẩn sibutramin và phenolphthalein Data File Name: TEA+30ppb 01 Quantitation Mass (m/z) $280.183->125.058 \mathrm{mz}$

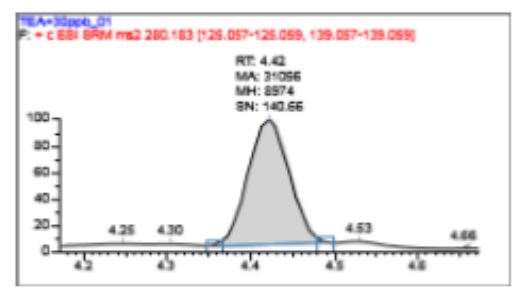

Compound

Ratio ion

RT

Amount

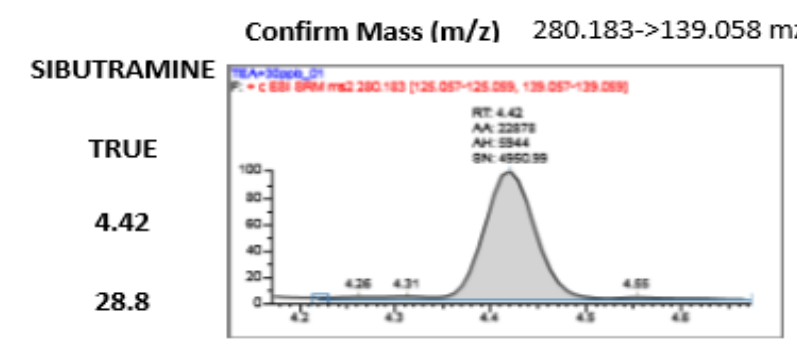

Master method:

Sibutramine PHENOLTHALEIN CTM Data File Name: TEA+30ppb_01

Quantitation Mass (m/z) 319.213->225.000 mz

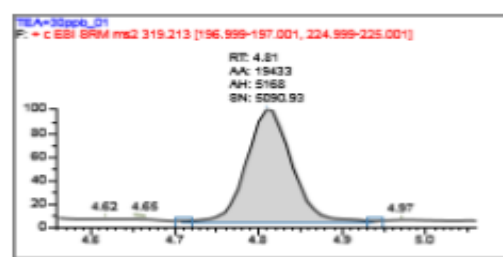

Compound

Ratio ion

RT

Amount

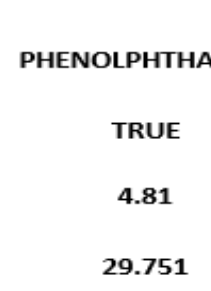

Confirm Mass $(\mathrm{m} / \mathrm{z}) \quad 319.213->197.000 \mathrm{mz}$

Hinh 3.3.3 c. Sắc ký đồ mẫu trà giảm cân thêm $30 \mu \mathrm{g} / \mathrm{kg}$ chuẩn sibutramin và phenolphthalein

\section{4. Ứng dụng phân tích thực phẩm giảm cân tại trung tâm dịch vụ phân tích thành phố Hồ Chí Minh}

Sau khi thẩm định, phương pháp phân tích được áp dụng để phân tích sibutramin và phenolphthalein trong một số mẫu thực phẩm giảm cân bao gồm trà, cà phê, sữa giảm cân. Kết quả 46 mẫu (03 - 08/2019) không phát hiện thấy 02 chất sibutramin và phenolphthalein trong mẫu.

\section{KẾT LUẬN}

Phương pháp xác định sibutramin và phenolphthalein trong trà, cà phê, sữa giảm cân bằng kỹ thuật UHPLC-MS/MS kết hợp với việc xử lý mẫu bằng kỹ thuật QuEChERS đạt được độ nhạy, độ chọn lọc và độ chính xác cao phù hợp theo tiêu chuẩn của AOAC (2016), $\mathrm{SANCO} / 12571 / 2013$. Phương pháp có thể được ứng dụng để đánh giá sibutramin và phenolphthalein trong trà, cà phê, sữa giảm cân góp phần kiểm soát chặt chẽ vấn đề an toàn vệ sinh thực phẩm nhằm hướng tới môi trường thực phẩm an toàn cho người tiêu dùng.

Trong thời gian sắp tới chúng tôi tiếp tục nghiên cứu các chất chuyển hóa sibutramin như $\mathrm{N}$-des methyl sibutramin (DSB) and N-di desmethyl sibutramin (DDSB). 


\section{TÀI LIẸU THAM KHẢO}

1. Nguyen Hong Vu, 2 chất cấm có trong trà giảm cân Golean Detox nguy hiểm thế nào, Báo sức khỏe đời sống, (2019).

2. Wang, D., Man, R., Shu, M., Liu, H., Gao, Y., \& Luan, F. (2016), "Detection of sibutramine and phenolphthalein in functional foods using capillary electrophoresis", Analytical Meth ods, 8 (3), 621 - 626.

3. Kim, J. Y., Park, H. J., Kim, J. W., Lee, J. H., Heo, S., Yoon, C. Y., \& Cho, S. (2016), "Development and validation of UPLC and LC-MS/MS methods for the simultaneous de termination of anti-obesity drugs in foods and dietary supplements", Archives of pharmacal research, 39 (1), 103 - 114.

4. Paíga, P., Rodrigues, M. J., Correia, M., Amaral, J. S., Oliveira, M. B. P., \& Delerue-Matos, C. (2017), "Analysis of pharmaceutical adulterants in plant food supplements by UHPLCMS/MS”, European Journal of Pharmaceutical Sciences, 99, 219 - 227.

5. Petkova-Gueorguieva, E., Ivanov, K., Gueorguiev, S., Mihaylova, A., Madzharov, V., \& Ivanova, S. (2018), "Detection of sibutramine in herbal food supplements by UHPLC/HRMS and UHPLC/MS-MS”, Biomedical Research, 29 (14), 3006 - 3009.

6. Guidelines for Standard Method Performance Requirements, AOAC Official Methods of Analysis (2016).

7. Guidance document on analytical quality control and validation procedures for pesticide residues analysis in food and feed, SANCO/12571/2013.

\section{Summary}

DETERMINATION OF SIBUTRAMINE AND PHENOLPHTHALEIN IN WEIGHT-LOSS TEA, COFFEE, AND MILK BY ULTRA-PERFORMANCE LIQUID CHROMATOGRAPHY MASS SPECTROMETRY UPLC- MS/MS

\section{Truong Van Than, Nguyen Thanh Duy*, Ly Tuan Kiet Hoang Ngoc Vinh, Chu Van Hai}

Center of Analytical Services and Experimentation Ho Chi Minh City

A rapid, simply and effective method using ultra-performance liquid chromatography coupled to mass spectrometry (MS) detection was developed in order to determine sibutramine and phenolphthalein in weight-loss tea, coffee, and milk. QuEChERS technique was used for cleaning-up sample matrices. The extracts were separated on C18 LC columns using the gradient elution of $0.1 \%$ formic acid in distilled water and acetonitrile. The recoveries at the concentration of $50 \mu \mathrm{g} / \mathrm{kg}$ were from 90.1 to $105.8 \%$ (with $\mathrm{RSD}_{\mathrm{R}}$ of 4.04 $4.24 \%$ ), from 91.9 to $100.2 \%$ (with $\mathrm{RSD}_{\mathrm{R}}$ of $3.20-4.71 \%$ ), and from 86.5 to $100.5 \%$ (with $\mathrm{RSD}_{\mathrm{R}}$ of $5.17-5.49 \%$ ) for weight-loss tea, coffee, and milk, respectively. Method quantitation limits were of $30 \mu \mathrm{g} / \mathrm{kg}$.

Keywords: Sibutramine, phenolphthalein, dietary supplements, ultra-performance liquid chromatography mass spectrometry UPLC-MS/MS. 